\title{
On the possibility of constraining cosmic topology from the celestial distribution of astronomical objects (Research Note)
}

\author{
H. Fujii and Y. Yoshii \\ Institute of Astronomy, School of Science, University of Tokyo, 2-21-1, Osawa, Mitaka, 181-0015 Tokyo, Japan \\ e-mail: hfujii@ioa.s.u-tokyo.ac.jp
}

Received 4 April 2011 / Accepted 11 May 2011

\begin{abstract}
We present a method of constraining cosmic topology from the distribution of astronomical objects projected on the celestial sphere. This is an extension of the 3D method we introduced in 2011 of searching for two pairs of observed objects (quadruplet) linked by a holonomy; i.e., the method we present here is to search for a pair of celestial sphere $n$-tuplets for $n \geq 3$. We find, however, that this method is impractical for application in realistic situations due to the small signal-to-noise ratio. We conclude therefore that it is unrealistic to constrain the topology of the Universe from the celestial distribution, and the 3D catalogs are necessary for the purpose.
\end{abstract}

Key words. cosmology: theory - large scale structure of Universe

\section{Introduction}

The shape of space is one of the most important themes in modern cosmology. Together with the assumption of the cosmological principle, Einstein's General Relativity connects a curvature of space with the average energy density of the universe, and recent observations suggest a flat $\Lambda$-CDM universe (e.g., $\Omega_{\text {tot }}=1.0050_{-0.0061}^{+0.0060}$ from $W M A P+\mathrm{BAO}+\mathrm{SN}$ data, by Hinshaw et al. 2009).

However, while General Relativity describes local geometry (curvature), it predicts nothing about the overall property of space, namely, the topology of the universe. There is no evidence for the general assumption that out Universe is simply connected; it can be multiconnected. A multiconnected space with a nontrivial topology can be imagined as a $2 K$-polyhedron, called the Dirichlet domain, whose $K$ pairs of faces are glued mathematically by holonomies. An object passing through one face immediately returns through the glued face. As a result, multiple images of single objects, often referred to as "ghosts", appear like those in a kaleidoscope (for details, see, e.g., Lachièze-Rey and Luminet 1995).

Many methods for constraining cosmic topology are based on this prediction, such as the circles-in-the-sky method (Cornish et al. 1998) that is to search for intersections of the last-scattering surface and the faces of our Dirichlet domain. They are circles with the same temperature fluctuation pattern in the CMB map, because they are copies of a physically identical region. Various authors have searched for matched circles using the WMAP data and obtained diverse results. For example, Aurich (2008) finds a hint of a 3-torus topology, while Roukema et al. (2008) finds a signature of a Poincaré dodecahedral space topology. In contrast to them, Cornish et al. (2004), Key et al. (2007), and Bielewicz \& Banday (2011) have found no topological signatures and obtained the lower limit of the size of our Universe. The most recent constraint is $\sim 27.9$ Gpc by Bielewicz \& Banday (2011).
These disagreements suggest that there are methodological problems and bring our interests to the 3D methods, i.e., those that are to use distributions of galaxies, galaxy clusters, or active galactic nuclei.

We used the 3D methods to search for unusual positional patterns of objects in a given astronomical catalog, which are caused by topological copies (e.g., Lehoucq et al. 1996; Roukema 1996; Uzan et al. 1999; Marecki et al. 2005; Fujii \& Yoshii 2011a). For example, if we live in a 3-torus universe, a pair separation histogram (PSH) of a given catalog will show sharp spikes at the separations corresponding to its fundamental length (Lehoucq et al. 1996). Fujii \& Yoshii (2011a) introduced a new method that is much more sensitive to topological signatures than the prior ones. This method can apply to any of 17 multiconnected Euclidean spaces, even if the whole universe is comparable to the observed region in size, i.e., in a case where there are only a few topological ghosts.

Our 3D method and others all require spectroscopical observations to calculate the radial distances of objects. A wide field spectroscopic survey is usually difficult, especially for the high-redshift objects in which we are most interested. Mainly to explore CMB data, Bernui \& Villela (2006) have extended the PSH method to the pair angular separation histogram (PASH) method, which does not require spectroscopic data when applied to astronomical objects. Their method, however, is so insensitive to topological signatures that the signal does not appear unless we average a number of different, but statistically equivalent PASHs, which is almost impossible in practice.

A significant number of high-redshift objects (galaxies or quasars) have recently been found, though most of them have not been spectroscopically observed yet. This is a good point in time to examine whether these objects are usable for constraining cosmic topology or not. In Sect. 2, we introduce another 2D method to constrain cosmic topology from the distribution 
of objects projected on the celestial sphere, which is an extension of the 3D method of Fujii \& Yoshii (2011a). In Sect. 3, we show that the method is applicable in principle, but in a realistic situation it is no longer useful, similar to that of Bernui \& Villela (2006). We conclude therefore that spectroscopically observed 3D catalogs are necessary, in order to reveal the topology of the Universe from the distribution of astronomical objects. Throughout the paper we consider flat universes with zero curvature.

\section{Method}

In this section we describe the methodology for judging whether a given pair of $n$-tuplets ( $2 n$-tuplet) are linked by some holonomy. Our assumption is that the universe has zero curvature (Euclidean geometry) and that the distances from us to the $2 n$ objects are unknown, while their celestial positions are known.

A convenient way of writing the holonomies is to use a 4D coordinate system $(w, x, y, z)$ where the simply connected 3 -Euclidean space is represented as a hyperplane $w=1$ (see, e.g., Fujii \& Yoshii 2011a). Then every holonomy $\gamma$ in a flat universe can be written as a 4D matrix, $\gamma=\gamma_{\mathrm{T}} \gamma_{\mathrm{NT}}$, where $\gamma_{\mathrm{T}}$ and $\gamma_{\mathrm{NT}}$ are a translational part and a nontranslational part, respectively. If a pair of $n$-tuplets $\left(\boldsymbol{x}_{1}, \cdots, \boldsymbol{x}_{n}\right)$ and $\left(\boldsymbol{x}_{1}^{\prime}, \cdots, \boldsymbol{x}_{n}^{\prime}\right)$ are linked by $\gamma$, we have

$\boldsymbol{x}_{1}^{\prime}=\gamma \boldsymbol{x}_{1}=\gamma_{\mathrm{T}} \gamma_{\mathrm{NT}} \boldsymbol{x}_{1}=\gamma_{\mathrm{NT}} \boldsymbol{x}_{1}+\boldsymbol{L}$,

$\boldsymbol{x}_{n}^{\prime}=\gamma \boldsymbol{x}_{n}=\gamma_{\mathrm{T}} \gamma_{\mathrm{NT}} \boldsymbol{x}_{n}=\gamma_{\mathrm{NT}} \boldsymbol{x}_{n}+\boldsymbol{L}$,

where $\boldsymbol{L}$ is the translational vector. A vector $\boldsymbol{X}$ is $4 \mathrm{D}, \boldsymbol{X}=$ $(1, X, Y, Z)$, but the 3D part $(X, Y, Z)$ is important. We know all the mathematical possibilities for $\gamma_{\mathrm{NT}}$ (an identity, an $n$th turn rotation for $n=2,3,4$, or 6 , or a reflection), so these $3 n$ equations have $2 n+3$ unknown quantities: the distances from us to the $2 n$ objects and $\boldsymbol{L}$. If the distances are known from spectroscopic observation, then the unknown quantities are just three, $\boldsymbol{L}=\left(L_{1}, L_{2}, L_{3}\right)$, so we derive $3 n-3$ conditions as we did for $n=2$ in Fujii \& Yoshii (2011a). In that work we introduced a new method of searching for quadruplets that satisfy the condition

$\boldsymbol{x}_{1}^{\prime}-\boldsymbol{x}_{2}^{\prime}=\gamma_{\mathrm{NT}}\left(\boldsymbol{x}_{1}-\boldsymbol{x}_{2}\right)$,

and showed that the method is extremely sensitive to topological signatures in a given catalog.

In the case considered here, however, we do not know the distances and have to take another way. For this, we first denote the $3 \mathrm{D}$ positions of objects as follows:

$\boldsymbol{x}=\left(\begin{array}{c}x \\ y \\ z\end{array}\right)=\left(\begin{array}{c}r \hat{x} \\ r \hat{y} \\ r \hat{z}\end{array}\right)=r \boldsymbol{R}$,

where $r$ is the unknown distance from us to the object, and the vector $\boldsymbol{R}=(\hat{x}, \hat{y}, \hat{z})$ represents the known celestial position such that $\hat{x}^{2}+\hat{y}^{2}+\hat{z}^{2}=1$. For the simplest case where $n=3$ and $\gamma_{\mathrm{NT}}=i d$, i.e., $\gamma$ is a translation, the following relations hold by eliminating $\boldsymbol{L}$ :

$r_{1} \boldsymbol{R}_{1}-r_{2} \boldsymbol{R}_{2}=r_{1}^{\prime} \boldsymbol{R}_{1}^{\prime}-r_{2}^{\prime} \boldsymbol{R}_{2}^{\prime}$,

$r_{1} \boldsymbol{R}_{1}-r_{3} \boldsymbol{R}_{3}=r_{1}^{\prime} \boldsymbol{R}_{1}^{\prime}-r_{3}^{\prime} \boldsymbol{R}_{3}^{\prime}$.
Then eliminating $r_{2}, r_{3}, r_{2}^{\prime}$, and $r_{3}^{\prime}$ from these equations gives

$$
\begin{aligned}
& {\left[\left(\boldsymbol{R}_{1} \times \boldsymbol{R}_{2}\right)_{z} \hat{z}_{2}^{\prime}+\left(\boldsymbol{R}_{2} \times \boldsymbol{R}_{2}^{\prime}\right)_{z} \hat{z}_{1}+\left(\boldsymbol{R}_{2}^{\prime} \times \boldsymbol{R}_{1}\right)_{z} \hat{z}_{2}\right] r_{1}} \\
& +\left[\left(\boldsymbol{R}_{1}^{\prime} \times \boldsymbol{R}_{2}^{\prime}\right)_{z} \hat{z}_{2}+\left(\boldsymbol{R}_{2}^{\prime} \times \boldsymbol{R}_{2}\right)_{z} \hat{z}_{1}^{\prime}+\left(\boldsymbol{R}_{2} \times \boldsymbol{R}_{1}^{\prime}\right)_{z} \hat{z}_{2}^{\prime}\right] r_{1}^{\prime}=0, \\
& {\left[\left(\boldsymbol{R}_{1} \times \boldsymbol{R}_{3}\right)_{z} \hat{z}_{3}^{\prime}+\left(\boldsymbol{R}_{3} \times \boldsymbol{R}_{3}^{\prime}\right)_{z} \hat{z}_{1}+\left(\boldsymbol{R}_{3}^{\prime} \times \boldsymbol{R}_{1}\right)_{z} \hat{z}_{3}\right] r_{1}} \\
& +\left[\left(\boldsymbol{R}_{1}^{\prime} \times \boldsymbol{R}_{3}^{\prime}\right)_{z} \hat{z}_{3}+\left(\boldsymbol{R}_{3}^{\prime} \times \boldsymbol{R}_{3}\right)_{z} \hat{z}_{1}^{\prime}+\left(\boldsymbol{R}_{3} \times \boldsymbol{R}_{1}^{\prime}\right)_{z} \hat{z}_{3}^{\prime}\right] r_{1}^{\prime}=0 .
\end{aligned}
$$

These are simultaneous equations for $r_{1}, r_{1}^{\prime}$ and should have a solution $\left(r_{1}, r_{1}^{\prime}\right) \neq(0,0)$, so the following relation must hold:

$A D-B C=0$,

where

$A=\left(\boldsymbol{R}_{1} \times \boldsymbol{R}_{2}\right)_{z} \hat{z}_{2}^{\prime}+\left(\boldsymbol{R}_{2} \times \boldsymbol{R}_{2}^{\prime}\right)_{z} \hat{z}_{1}+\left(\boldsymbol{R}_{2}^{\prime} \times \boldsymbol{R}_{1}\right)_{z} \hat{z}_{2}$,

$B=\left(\boldsymbol{R}_{1}^{\prime} \times \boldsymbol{R}_{2}^{\prime}\right)_{z} \hat{z}_{2}+\left(\boldsymbol{R}_{2}^{\prime} \times \boldsymbol{R}_{2}\right)_{z} \hat{z}_{1}^{\prime}+\left(\boldsymbol{R}_{2} \times \boldsymbol{R}_{1}^{\prime}\right)_{z} \hat{z}_{2}^{\prime}$,

$C=\left(\boldsymbol{R}_{1} \times \boldsymbol{R}_{3}\right)_{z} \hat{z}_{3}^{\prime}+\left(\boldsymbol{R}_{3} \times \boldsymbol{R}_{3}^{\prime}\right)_{z} \hat{z}_{1}+\left(\boldsymbol{R}_{3}^{\prime} \times \boldsymbol{R}_{1}\right)_{z} \hat{z}_{3}$,

$D=\left(\boldsymbol{R}_{1}^{\prime} \times \boldsymbol{R}_{3}^{\prime}\right)_{z} \hat{z}_{3}+\left(\boldsymbol{R}_{3}^{\prime} \times \boldsymbol{R}_{3}\right)_{z} \hat{z}_{1}^{\prime}+\left(\boldsymbol{R}_{3} \times \boldsymbol{R}_{1}^{\prime}\right)_{z} \hat{z}_{3}^{\prime}$.

This relation is written by the celestial positions of the objects, so spectroscopic observations are not needed to use it. Though we have considered the special case here that $\gamma$ is a translation, $A, B, C$, and $D$ for other cases are calculated similarly. If $\gamma$ is a half-turn corkscrew motion or a glide reflection,

$$
\begin{aligned}
& A=-\left(\boldsymbol{R}_{1} \times \boldsymbol{R}_{2}\right)_{z} \hat{z}_{2}^{\prime}+\left(\boldsymbol{R}_{2} \times \boldsymbol{R}_{2}^{\prime}\right)_{z} \hat{z}_{1}+\left(\boldsymbol{R}_{2}^{\prime} \times \boldsymbol{R}_{1}\right)_{z} \hat{z}_{2}, \\
& B=\left(\boldsymbol{R}_{1}^{\prime} \times \boldsymbol{R}_{2}^{\prime}\right)_{z} \hat{z}_{2}-\left(\boldsymbol{R}_{2}^{\prime} \times \boldsymbol{R}_{2}\right)_{z} \hat{z}_{1}^{\prime}-\left(\boldsymbol{R}_{2} \times \boldsymbol{R}_{1}^{\prime}\right)_{z} \hat{z}_{2}^{\prime}, \\
& C=-\left(\boldsymbol{R}_{1} \times \boldsymbol{R}_{3}\right)_{z} \hat{z}_{3}^{\prime}+\left(\boldsymbol{R}_{3} \times \boldsymbol{R}_{3}^{\prime}\right)_{z} \hat{z}_{1}+\left(\boldsymbol{R}_{3}^{\prime} \times \boldsymbol{R}_{1}\right)_{z} \hat{z}_{3}, \\
& D=\left(\boldsymbol{R}_{1}^{\prime} \times \boldsymbol{R}_{3}^{\prime}\right)_{z} \hat{z}_{3}-\left(\boldsymbol{R}_{3}^{\prime} \times \boldsymbol{R}_{3}\right)_{z} \hat{z}_{1}^{\prime}-\left(\boldsymbol{R}_{3} \times \boldsymbol{R}_{1}^{\prime}\right)_{z} \hat{z}_{3}^{\prime} .
\end{aligned}
$$

And if $\gamma$ is an $n$th turn corkscrew motion for $n=4,3$, or 6 , then

$$
\begin{aligned}
A= & \left(\boldsymbol{R}_{1} \times \boldsymbol{R}_{2}\right)_{z} \hat{z}_{2}^{\prime}+\left\{\left(\boldsymbol{R}_{2} \times \boldsymbol{R}_{2}^{\prime}\right)_{z} \hat{z}_{1}+\left(\boldsymbol{R}_{2}^{\prime} \times \boldsymbol{R}_{1}\right)_{z} \hat{z}_{2}\right\} c \\
& +\left\{\boldsymbol{R}_{2}^{\prime} \times\left(\boldsymbol{R}_{2} \times \boldsymbol{R}_{1}\right)\right\}_{z} s, \\
B= & \left(\boldsymbol{R}_{1}^{\prime} \times \boldsymbol{R}_{2}^{\prime}\right)_{z} \hat{z}_{2}+\left\{\left(\boldsymbol{R}_{2}^{\prime} \times \boldsymbol{R}_{2}\right)_{z} \hat{z}_{1}^{\prime}+\left(\boldsymbol{R}_{2} \times \boldsymbol{R}_{1}^{\prime}\right)_{z} \hat{z}_{2}^{\prime}\right\} c \\
& -\left\{\boldsymbol{R}_{2} \times\left(\boldsymbol{R}_{2}^{\prime} \times \boldsymbol{R}_{1}^{\prime}\right)\right\}_{z} s, \\
C= & \left(\boldsymbol{R}_{1} \times \boldsymbol{R}_{3}\right)_{z} \hat{z}_{3}^{\prime}+\left\{\left(\boldsymbol{R}_{3} \times \boldsymbol{R}_{3}^{\prime}\right)_{z} \hat{z}_{1}+\left(\boldsymbol{R}_{3}^{\prime} \times \boldsymbol{R}_{1}\right)_{z} \hat{z}_{3}\right\} c \\
& +\left\{\boldsymbol{R}_{3}^{\prime} \times\left(\boldsymbol{R}_{3} \times \boldsymbol{R}_{1}\right)\right\}_{z} s, \\
D= & \left(\boldsymbol{R}_{1}^{\prime} \times \boldsymbol{R}_{3}^{\prime}\right)_{z} \hat{z}_{3}+\left\{\left(\boldsymbol{R}_{3}^{\prime} \times \boldsymbol{R}_{3}\right)_{z} \hat{z}_{1}^{\prime}+\left(\boldsymbol{R}_{3} \times \boldsymbol{R}_{1}^{\prime}\right)_{z} \hat{z}_{3}^{\prime}\right\} c \\
& -\left\{\boldsymbol{R}_{3} \times\left(\boldsymbol{R}_{3}^{\prime} \times \boldsymbol{R}_{1}^{\prime}\right)\right\}_{z} s,
\end{aligned}
$$

where $c=\cos (2 \pi / n)$ and $s=\sin (2 \pi / n)$. The cases for $n=1$ and $n=2$ correspond to translation and half-turn corkscrew motion, respectively.

Given a catalog with $N$ objects, we count the number of sextuplets ( $2 n$-tuplets for $n=3$ ) satisfying the condition of $A D-B C=0$ for each type of holonomies, but within a chosen tolerance $\varepsilon$, i.e., $|A D-B C|<\varepsilon$. The number of such sextuplets will be greater for a multiconnected space than is stochastically expected for a simply connected one. We assign each object $\boldsymbol{x}_{i}$ an integer $s_{i}$, the number of sextuplets that satisfy the condition $|A D-B C|<\varepsilon$ and also include $\boldsymbol{x}_{i}$ as one of their members. An $s_{i}$-histogram for a multiconnected space will have some bumps in the large- $s_{i}$ region, since topological copies contribute to the signal more frequently.

Similar calculations can be done for $n \geq 4$ where we have additional conditions; however, the calculation time is roughly proportional to ${ }_{N} C_{n}=N ! /(N-n) ! n !$, and the calculation for a large $n$ is unrealistically time-consuming. In the next section we show the results for $n=3$, the minimum value above which this method can apply, and discuss the possibility that we can constrain the topology of the Universe by the method. 
Table 1. Results for various types of holonomies.

\begin{tabular}{l|c|c|c|c}
\hline \hline Holonomy type & $\begin{array}{c}\text { Number of } \\
\text { quasars }\end{array}$ & $\begin{array}{c}\text { Number of } \\
\text { ghosts }\end{array}$ & $\begin{array}{c}\text { Topological } \\
\text { index } S_{\text {mult }}\end{array}$ & $\begin{array}{c}\text { Topological } \\
\text { index } S_{\text {simp }}\end{array}$ \\
\hline Translation & 57 & 14 & 224 & 116 \\
Half-turn corkscrew motion & 60 & 20 & 733 & 175 \\
Quarter-turn corkscrew motion & 58 & 16 & 186 & 138 \\
Third-turn corkscrew motion & 57 & 14 & 318 & 235 \\
Sixth-turn corkscrew motion & 59 & 18 & 372 & 291 \\
Glide reflection & 58 & 16 & 359 & 153 \\
\hline
\end{tabular}

Notes. Topological index $S_{\text {mult }}$ is the number of sextuplets satisfying $|A D-B C|<10^{-8}$ for a multiconnected space, and $S_{\text {simp }}$ is for the simply connected one.

\section{Simulations and discussions}

In order to understand the applicability of the method described in the previous section, we generated toy catalogs in simply and multiconnected Euclidean spaces. As multiconnected spaces, we considered the following six cases:

- one with a pair of translations;

- four with a pair of $n$th turn corkscrew motions for $n=2,3,4$, and 6;

- and one with a pair of glide reflections.

For each simulation, the observed region is a unit sphere centered at the observer's position, and the translational distance is $L=|\boldsymbol{L}|=1.4$, which implies that $1-\int_{-0.7}^{0.7} \pi\left(1-x^{2}\right) \mathrm{d} x / \frac{4 \pi}{3} \sim 12 \%$ of the observed region is a copy of some part of the "first" copy of the universe. Now consider $L$ to be $c / H_{0} \simeq 4.2 \mathrm{Gpc}$, where $c$ and $H_{0}$ are the speed of light and the Hubble parameter, respectively. With this example scale, the radius of the observed region is $\sim 3.0 \mathrm{Gpc}$, corresponding to $z \sim 0.9$. The effects of global inhomogeneity that had been investigated in detail in Fujii \& Yoshii (2011a) were not considered here. The axes for the $n$th turn corkscrew motions and the reflectional plane for the glide reflections pass through the observer, and we chose the correct coordinate systems.

Our toy catalogs were made as follows. We uniformly distributed 50 real objects, and then their copies were generated for each multiconnected space. The total number of objects $N$ (50 real ones plus their copies) is different from simulation to simulation, since some of the copies were beyond the observed region. Next, another catalog in the simply connected space with the same number of objects was made for comparison. All objects were projected on the celestial sphere, and sextuplets satisfying the condition $|A D-B C|<\varepsilon$ were searched for.

First, we succeeded in detecting topological signal with the choice of $\varepsilon=10^{-8}$. The results are given in Table 1 . The number of sextuplets that satisfy the condition of $|A D-B C|<\varepsilon$, for each case, is given as "topological index". (We had normalized the topological indices with respect to the total number of quadruplets in Fujii \& Yoshii 2011a, but not here.) As expected, the topological index for a multiconnected space is larger than for the simply connected one, which successfully distinguishes the two topologies. The difference can be seen more clearly in $s_{i}$-histograms as shown in Fig. 1 . Some bumps are seen in the large- $s_{i}$ regions for multiconnected cases, because of the topological copies that contribute to the signal more frequently than a stochastic expectation.

These results show that this $2 \mathrm{D}$ method in principle does constrain the topology of the Universe; however, the practical
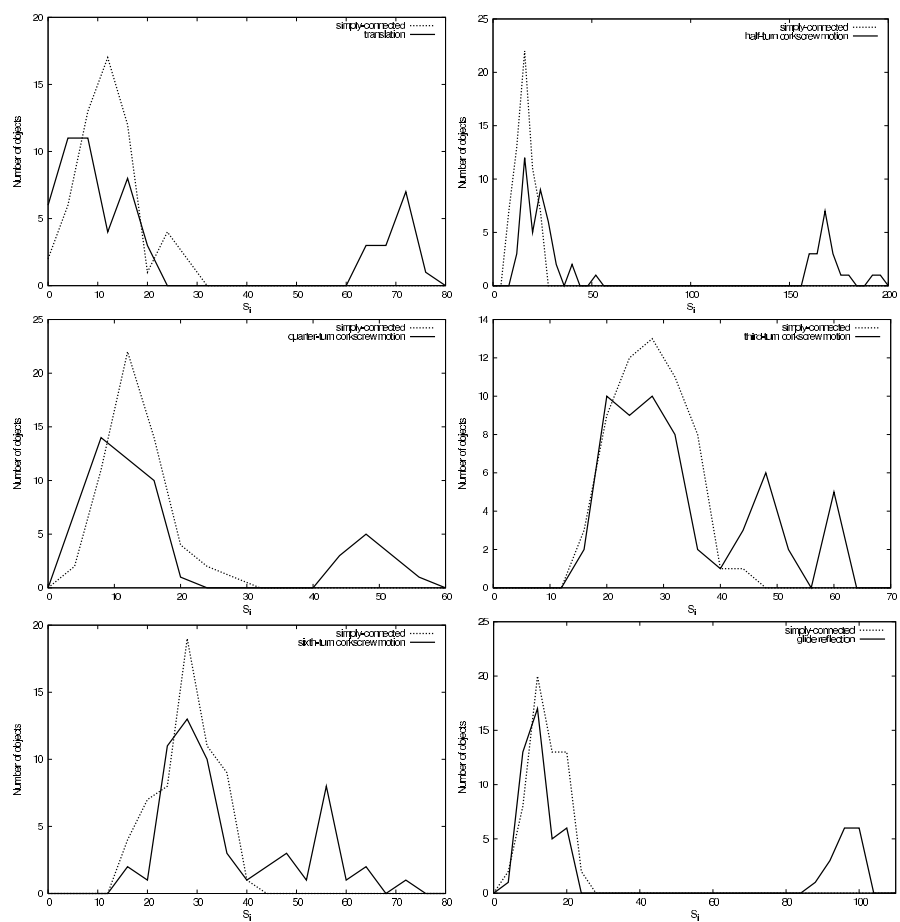

Fig. 1. The $s_{i}$-histograms for various types of holonomies: translation (top left), half-turn corkscrew motion (top right), quarter-turn corkscrew motion (mid left), third-turn corkscrew motion (mid right), sixth-turn corkscrew motion (lower left), and glide reflection (lower right). Bumps constituted by topological copies for these types are seen in the large $s_{i}$ regions for multiconnected spaces.

application of the method is not very straightforward since the condition considered here is optimistic and somewhat arbitrary:

- The tolerance as small as $\varepsilon=10^{-8}$ cannot be chosen when correctly considering the limited resolution, e.g., with the typical ground-based optical instruments, $\lesssim 1$ arcsec, which implies uncertainties in $\varepsilon$ on the order of $10^{-6}$. Moreover, even if we have an ideal instrument, owing to the peculiar velocity, the quantity $|A D-B C|$ will be larger than $10^{-8}$ even for a really topological sextuplet. For example, the typical peculiar velocity of $v=500 \mathrm{~km} \mathrm{~s}^{-1}$ and the time lag of $10^{8} \mathrm{yr}$ (corresponding to an upper limit to quasar lifetimes) implies that $\varepsilon$ should be more than $\sim 10^{-5}$ at the redshift $z \sim 5$. These large tolerances drastically enhance the stochastic noise.

- The ratio of the number of topological ghosts to the total number of objects $(N)$ is large here, $\sim 12-17 \%$. In reality, however, it can be much less than this because of a tiny number of ghosts, a large number of objects, or both, so 
the topological signal will be hidden by the stochastic noise: roughly speaking, the former scales as $N^{3}$, the latter as $N^{6}$.

- Each simulation here took several minutes using an ordinary personal computer. Howeover, calculations for a realistic value of $N \sim 10^{3}$ takes an extremely long time, which is roughly proportional to $N C_{3}=N(N-1)(N-2) / 6$.

For example, with the choice of $\varepsilon=10^{-5}$ and $N \sim 60$, the stochastic noise is on the order of $10^{5}$, completely hiding the topological signal of $10^{2}$. The situation gets even worse for a larger $N$.

Thus the method will be impractical in realistic situations due to a low signal-to-noise ratio. One way to overcome the problem may be using $n \geq 4$, rather than $n=3$. For a given pair of $n$-tuplets to be linked by a holonomy, there are $n-2$ conditions to be satisfied, thus we have $n-2$ filters. The possibility $P(n)$ that a nontopological $2 n$-tuplet passes through all the filters by chance monotonically decreases with $n$. Therefore, the stochastic noise $P(n) \times{ }_{N} C_{n}$, can be suppressed if we choose large enough $n$. However, as mentioned in the previous section, the calculation time roughly scales as ${ }_{N} C_{n}=N ! /(N-n) ! n !$, and calculations for such a large $n$ are extremely time-consuming.

Considering these circumstances, we must conclude that it is unrealistic to constrain cosmic topology from the celestial $2 \mathrm{D}$ distribution of objects. For the practical purpose, we instead need the 3D catalogs of cosmic objects obtained by spectroscopic observations. The new crystallographic method introduced by Fujii \& Yoshii (2011a) can be applied to such catalogs, and it provides a knowledge about the shape of the Universe.
More than ever this will strengthen the motivation for promoting a large-scale spectroscopy survey of high-redshift objects.

Acknowledgements. We thank T. Minezaki, T. Tsujimoto, T. Yamagata, Y. Sakata, T. Kakehata, and K. Hattori for useful discussions and suggestions.

\section{References}

Aurich, R. 2008, Class. Quantum Grav., 25, 225017

Aurich, R., Janzer, H. S., Lustig, S., \& Steiner, F. 2008, Class. Quantum Grav., 25,125006

Bernui, A., \& Villela, T. 2006, A\&A, 445, 795

Bielewicz, P., \& Banday, A. J. 2011, MNRAS, 412, 2104

Cornish, N. J., Spergel, D. N., \& Starkman, G. D. 1998, Class. Quantum Grav., 15,2657

Cornish, N. J., Spergel, D. N., Starkman, G. D., \& Komatsu, E. 2004, Phys. Rev. Lett., 92, 201302

Fujii, H., \& Yoshii, Y. 2011, A\&A, 529, A121

Hinshaw, G., Weiland, J. L., Hill, R. S., et al. 2009, ApJS, 180, 225

Key, J. S., Cornish, N. J., Spergel, D. N., \& Starkman, G. D. 2007, Phys. Rev. D, 75,084034

Lachieze-Rey, M., \& Luminet, J. 1995, Phys. Rep., 254, 135

Lehoucq, R., Lachieze-Rey, M., \& Luminet, J. P. 1996, A\&A, 313, 339

Lehoucq, R., Uzan, J., \& Luminet, J. 2000, A\&A, 363, 1

Lew, B., \& Roukema, B. 2008, A\&A, 482, 747

Marecki, A., Roukema, B. F., \& Bajtlik, S. 2005, A\&A, 435, 427

Mota, B., Reboucas, M. J., \& Tavakol, R. 2010, Phys. Rev. D, 81, 103516

Roukema, B. F. 1996, MNRAS, 283, 1147

Roukema, B. F., Lew, B., Cechowska, M., Marecki, A., \& Bajtlik, S. 2004, A\&A, 423,821

Roukema, B. F., Buliński, Z., \& Gaudin, N. E. 2008a, A\&A, 492, 657

Roukema, B. F., Buliński, Z., Szaniewska, A., \& Gaudin, N. E. 2008b, A\&A, 486,55

Uzan, J., Lehoucq, R., \& Luminet, J. 1999, A\&A, 351, 766 